\title{
Polo-like kinase 4: the odd one out of the family
}

\author{
James E Sillibourne, Michel Bornens*
}

\begin{abstract}
Polo-like kinase 4 (PLK4) is a unique member of the Polo-like family of kinases that shares little homology with its siblings and has an essential role in centriole duplication. The turn-over of this kinase must be strictly controlled to prevent centriole amplification. This is achieved, in part, by an autoregulatory mechanism, whereby PLK4 autophosphorylates residues in a PEST sequence located carboxy-terminal to its catalytic domain. Phosphorylated PLK4 is subsequently recognized by the SCF complex, ubiquitinylated and targeted to the proteasome for degradation. Recent data have also shown that active PLK4 is restricted to the centrosome, a mechanism that could serve to prevent aberrant centriole assembly elsewhere in the cell. While significant advances have been made in understanding how PLK4 is regulated it is certain that additional regulatory mechanisms exist to safeguard the fidelity of centriole duplication. Here, we overview past and present data discussing the regulation and functions of PLK4.
\end{abstract}

\section{The structure of PLK4}

PLK4 was initially identified in the mouse as a kinase sharing homology with Drosophila Polo kinase, S. cerevisiae CDC5 and murine Snk (it was subsequently named as Snk akin kinase (SAK) due to its homology with the latter) [1]. Recent evolutionary studies have shed light onto the origins of PLK4/SAK, which appears to have arisen through gene duplication and subsequent subfunctionalization [2,3]. Homologues of PLK4 are present in most opisthokonts (organisms that have a single posterior flagellum), with at least one exception, the nematode C. elegans. This organism has no direct homolog of PLK4 although the kinase zyg-1 has been proposed to be a functional equivalent because it is essential for centriole duplication in the worm [4]. Interestingly, zyg-1 shares closer homology to the centrosomal kinases NIMA and MPS1 than to C. elegans Polo-like kinases (PLK1-3) strongly suggesting that it did not arise through duplication of the PLK gene [2].

While the structure of PLK4, in terms of arrangement of its functional domains (Figure 1), is similar to that of the other members of the Polo-like kinase family there are several significant differences and consequently its sequence is more divergent in comparison [5]. Common to the PLKs is an amino-terminal catalytic domain, which contains the unique ATP-binding site Gly-X-Gly$\mathrm{X}$-Phe-Ala, as opposed to the Gly-X-Gly-X-X-Gly motif

\footnotetext{
* Correspondence: michel.bornens@curie.fi Institut Curie, UMR144, 26 rue d'Ulm, 75248, Paris Cedex 05, France commonly found in kinases [6-8]. Sequence homology of the catalytic domain is highest in the first three members of the PLK family, with PLK1 sharing 53 and 54\% identity with PLK2 and PLK3, respectively while it is lower for PLK4 which only shares $37 \%$ identity with PLK1 [9]. Predicting PLK4 phosphorylation sites has proven to be difficult because the kinase phosphorylates in a context-dependent manner, whereby residues surrounding the phosphorylation site influence the kinase's ability to phosphorylate it [10]. Three different groups have derived consensus phosphorylation motifs [9-11], all of which share some common elements (Table 1).

All members of the family of Polo-like kinases possess a characteristic Polo-box, a conserved 64 amino acid motif located at the carboxyl terminus of the protein, which not only dictates the substrate specificity of the kinase, but also regulates its function [12,13]. PLK1, 2 and 3 possess two Polo-box domains at their C-terminus, while PLK4 only has one [14]. In place of a second Polo-box, PLK4 possesses a larger crypto Polo-box domain that has weaker homology with the Polo-box domain $[14,15]$. The fact that PLK4 only possesses a single Polo-box has important implications for its regulation and substrate repertoire. PLKs 1 to 3 bind to proteins that have previously been phosphorylated via their tandem Polo-boxes, which form intramolecular heterodimers and recognize the sequence Ser-pSer/ pThr-Pro-X [13]. Polo-box dimerization and binding to the phospho-motif is thought to regulate the activity of the kinase by inducing a change in its conformation, 


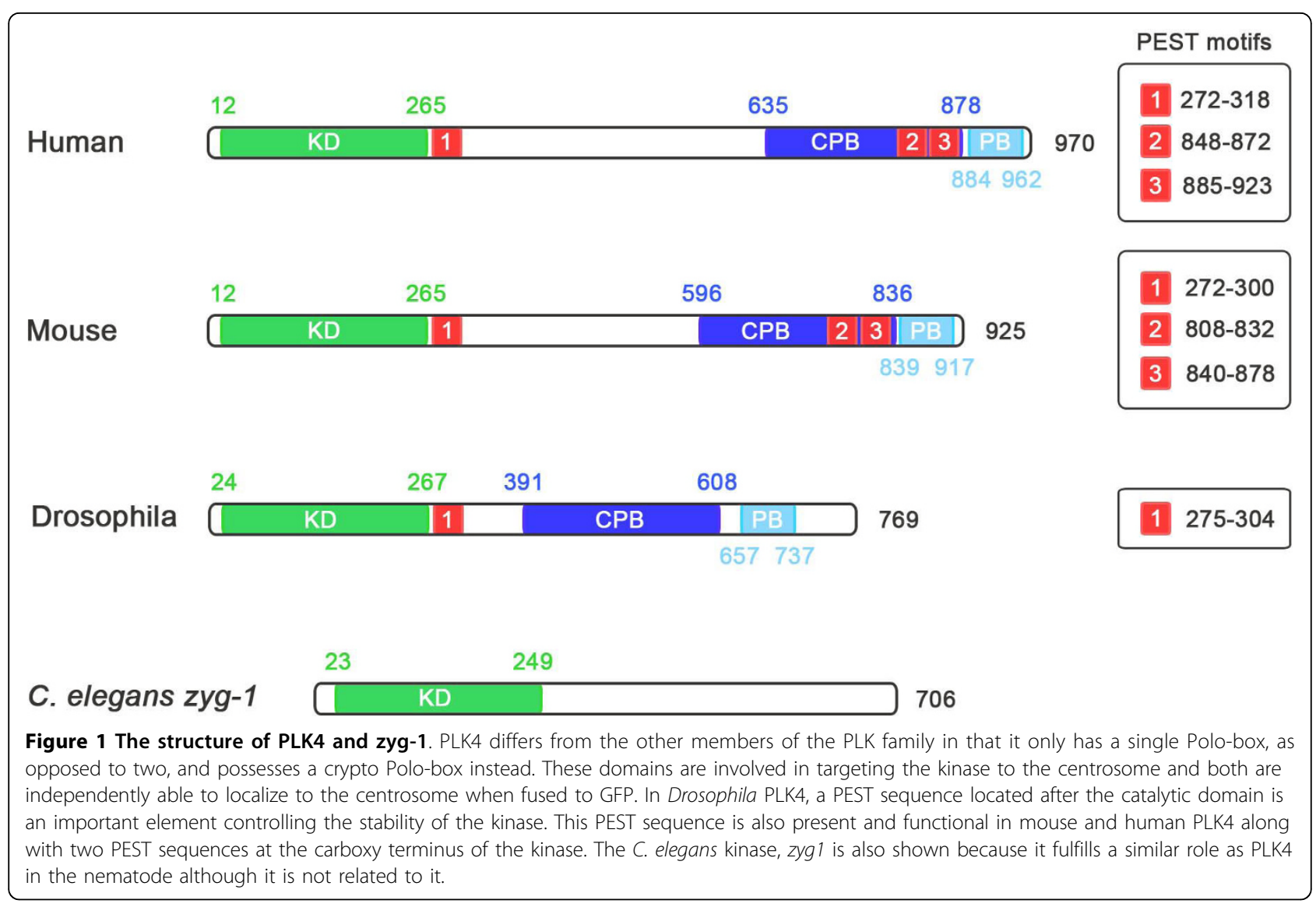

allowing the catalytic domain to have access to its substrate [13]. Because the Polo-box and crypto Polo-box of PLK4 do not form an intramolecular heterodimer, it has been suggested that PLK4 is not subject to the same form of regulation $[14,15]$. The PLK4 Polo-box does, however, homodimerize in an intermolecular manner and this may be involved in regulating PLK4 kinase activity [14]. The Polo-boxes of PLK1-3 are also important for targeting the kinases to particular subcellular sites and in this respect the Polo-box and crypto Polobox of PLK4 serve a similar function. Both are independently able to localize to centrosome, when expressed in fusion with EGFP [14], and only when both are removed from PLK4 does it fail to localize to centrosome and its function is suppressed $[14,16]$. This suggests that the Polo- and crypto Polo-boxes of PLK4 are protein-protein interaction domains responsible for targeting the kinase to the centrosome although the identities of their

Table 1 PLK4 phosphorylation motifs

\begin{tabular}{|c|c|c|c|c|c|c|c|c|}
\hline & -3 & -2 & -1 & $\mathrm{~S} / \mathrm{T}$ & 1 & 2 & 3 & 4 \\
\hline $\begin{array}{l}\text { Leung et al. } \\
\qquad(2007)\end{array}$ & - & Charged & $\begin{array}{l}\mathrm{I}, \mathrm{L} \text { and } \mathrm{V} \\
\text { unfavoured }\end{array}$ & & Hydrophobic (large) & $\begin{array}{l}\text { Hydrophobic } \\
\text { (large) }\end{array}$ & $x$ & $\begin{array}{l}\text { Charged } \\
\text { or P }\end{array}$ \\
\hline $\begin{array}{l}\text { Johnson et al. } \\
(2007)\end{array}$ & $\mathrm{R} / \mathrm{K}$ & $E / D$ & $x$ & & Hydrophobic/Y & Hydrophobic/Y & $x$ & $\mathrm{~S} / \mathrm{T} / \mathrm{A}$ \\
\hline $\begin{array}{l}\text { Sillibourne } \\
\text { et al. (2010) }\end{array}$ & $\begin{array}{l}\text { Aliphatic, hydrophobic or basic } \\
\text { (small to medium) }\end{array}$ & $x$ & $\begin{array}{l}\text { Large residues } \\
\text { unfavoured }\end{array}$ & & $\begin{array}{l}\text { Aliphatic (charged } \\
\text { residues unfavoured) }\end{array}$ & $\begin{array}{l}\text { Aromatic or } \\
\text { aliphatic (large) }\end{array}$ & $x$ & - \\
\hline
\end{tabular}

Three different groups have derived a PLK4 phosphorylation motif, while differences exist there are some common elements in all three. PLK4 has a preference for small to medium aliphatic or basic residues at the -3 position, aliphatic or charged residues at the +1 position and aromatic or large hydrophobic residues at the +2 position. The studies of Leung et al (2007) and Sillibourne et al (2010) show that large amino acids, in particular I, L and V, are unfavoured at the -1 position. There is a preference for a charged residue at the -2 position which is influenced by the residue in the +4 position and those outside of the motif. The interdependency between residues in the -2 and +4 positions, coupled with the influence of residues surrounding the phosphorylation motif, indicates that PLK4 is a context dependent kinase and renders the prediction of phosphorylation sites more difficult. Supporting this, it has been shown that a large number of predicted sites present in candidate PLK4 substrates, which fit the consensus phosphorylation motif well, are not phosphorylated by the kinase. All in all, this means that the identification of PLK4 substrates will remain a significant challenge for the future. 
binding partners remain to be discovered. The ability of the Polo-box and crypto Polo-box domains of PLK4 to bind to the centrosome could also be explained by the fact that both are able to self-associate with other domains within the kinase [14].

A further difference between PLK4 and the other PLKs is that it possesses a large central domain, which is conserved through evolution, although the function of this domain remains unknown [2].

PLK4 also possesses three PEST sequences, domains rich in proline $(P)$, aspartate $(D)$, glutamate $(E)$, serine $(\mathrm{S})$ and threonine $(\mathrm{T})$ residues, which govern protein stability $[1,17]$. The first PEST sequence is conserved, being present in many species including, $H$. sapiens, $M$. musculus, D. melanogaster, D. rerio and $X$. laevis $[18,19]$. The function of these sequences in regulating the turn-over of PLK4 will be discussed in more detail later.

\section{Functions of PLK4}

Studies carried out in knockout mice have demonstrated that PLK4 is essential for postgastrulative embryonic development and is required for mitotic progression [20]. PLK4-/- embryos arrest at stage E7.5 with increased numbers of apoptotic and late mitotic cells [20], while PLK4+/- embryos develop normally but have an increased incidence of spontaneous liver and lung cancers [21]. Partial hepatectomy experiments on PLK4 $+/$ - mice identified a defect in mitotic entry and exit, with cyclin B1 accumulation being delayed and prolonged for longer than normal [21]. Inspection of dividing hepatocytes from these mice showed that nearly one third had tripolar or tetrapolar spindles, which consequently led to the formation of disorganized liver tissue and an increased incidence of tumors [21]. Embryonic fibroblasts derived from PLK4+/- mice have supernumerary centrosomes, frequently undergo aberrant chromosome segregation and have a higher level of aneuploidy than wild-type mice [21].

At present, two mitotic PLK4 substrates have been identified: the phosphatase CDC25C [22] and the RhoA guanine exchange factor (GEF), Ect2 [23]. CDC25 was selected as a candidate PLK4 substrate based on the fact that this phosphatase is phosphorylated by PLK1 and PLK3 (raising the possibility that it is a common PLK substrate) and both CDC25C and PLK4 localize to the centrosome. Ect2 also seems to be a common PLK substrate, as it is phosphorylated by both PLK1 [24] and PLK4 [23]. Ect2 is a guanine exchange factor for the small GTPase RhoA and is required to activate it during cytokinesis to ensure correct positioning of the cleavage furrow [25]. Association of Ect2 to the central spindle is dependent upon PLK4 activity, as it fails to localize correctly in PLK4+/- MEFs. These cells frequently undergo cytokinetic failure because of the lack of Ect2 at the central spindle and insufficient RhoA activity [23].

\section{PLK4 and centriole duplication}

The centrosome duplicates once per cell cycle [26] and PLK4 plays an essential role in this process $[16,27]$. Over-expression of PLK4 in somatic cells results in the excessive formation of centrioles [16], the core structures of the centrosome, and in Drosophila oocytes the de novo formation of centrioles [28]. Conversely, depletion of PLK4 by RNAi prevents centriole duplication [27], causing mitotic defects and in some cell lines it can induce apoptosis [29].

A centrosome consists of two centrioles [26], barrelshaped microtubule-based structures, which are connected at their proximal ends by a flexible linker [30,31] (Figure 2). The two centrioles differ from one another, as one is slightly longer and possesses two sets of appendages at its distal end (sub-distal and distal appendages) [31,32]. This centriole is referred to as the mother and the other as the daughter centriole. The proximal ends of each centriole are surrounded by a matrix of proteins, referred to as pericentriolar material (PCM), which serves as a site of microtubule nucleation $[26,32]$. The PCM also serves to create environment favourable for the assembly of procentrioles [33], nascent centrioles, which form orthogonally from the existing centrioles [26,32,34]. Centriole duplication, similar to DNA replication is licensed to occur once per cell cycle $[35,36]$ and procentriole assembly starts at the G1/S border [34,37]. Procentriole assembly begins with the formation of a cartwheel structure to which microtubules are attached and elongated during the course of the cell cycle. The initial steps of procentriole assembly are dependent upon several proteins including SAS-6 [38], Cep135 [39,40], SAS-4 (CPAP) [41,42], $\gamma$-tubulin [43] and CP110 [44] as well as PLK4 [16,27]. At present, the centriolar substrates of PLK4 remain to be identified although SAS-6 is a possible candidate as work carried out in $C$. elegans has shown that zyg-1dependent phosphorylation of SAS-6 is required for procentriole formation [45]. Other factors are also required and include an array of kinases, such as PLK1 [46], PLK2 [47], cyclin-dependent kinase 2 (Cdk2)/cyclin $\mathrm{A} / \mathrm{E}[48,49]$ and Mps1 [50] as well as the phosphatase Cdc14B [51]. Later on in the cell cycle, during mitosis, procentriole elongation is completed [37] and proteins found in mature centrioles, such as the distal lumen protein hPOC5 [52], become incorporated into the procentriole structure. Throughout the duplication process, each procentriole remains closely associated with its parental centriole and this tight association (referred to as engagement) is a key aspect of the licensing mechanism $[34,35]$. It prevents centriole reduplication and it is 


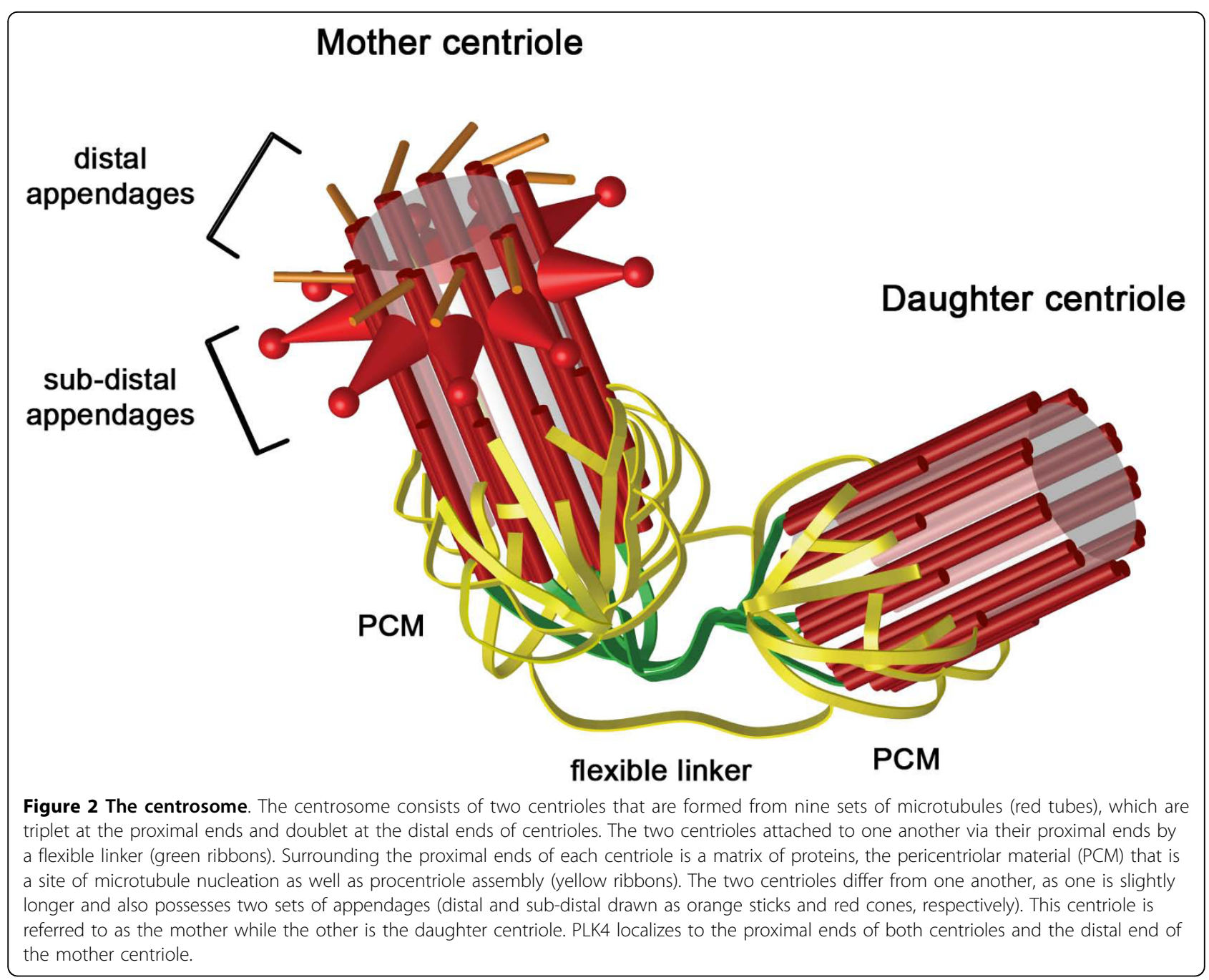

only during late mitosis that it is lost [53]. Centriole disengagement allows a new round of centriole duplication to occur in the next cell cycle and thereby acting as a licensing mechanism. Disengagement is dependent upon the activity of separase, a protease involved in breaking sister chromatid cohesion in mitosis and PLK1 [46].

Immunoelectron microscopy has shown that myctagged PLK4 localizes to the outer wall of centrioles and seems to be enriched at the proximal ends [43]. This localization is consistent with PLK4's role in centriole duplication because it is next the site of procentriole formation. However, PLK4 has also been observed, by immunofluorescent microscopy, at the distal end of the mother centriole close to the sub-distal and distal appendages [11]. The exact function of PLK4 at the distal end of the mother centriole remains to be elucidated, but it may be involved in centriole maturation including, centriole elongation and/or appendage assembly.
PLK4 abundance and activity during the cell cycle PLK4 abundance must be tightly controlled to ensure that centriole duplication goes according to plan, as either too little or too much of the kinase can have a deleterious effect upon the fidelity of centriole duplication. Too much PLK4, as demonstrated by over-expression of the kinase, overrides the centriole licensing mechanism and results in centriole amplification with multiple procentrioles forming around each parental centriole [16,27]. An insufficient amount of PLK4 may also give rise to the formation of abnormal centrioles and microtubule-based structures. In HCT116 cells microtubule-based $\gamma$-tubulin-containing structures lacking key centriolar components such as SAS-4/CPAP, SAS-6, and Cep135 have been observed [54]. These structures are commonly formed of microtubule bundles and some resemble centrioles, but lack a large number of centrosomal proteins and are unable to nucleate microtubules. Importantly, the incidence of these 
structures is reduced upon expression of PLK4 suggesting that there formation is due to insufficient kinase activity. Supporting these data, embryonic fibroblasts derived from PLK4 heterozygous mice exhibit an increased incidence of supernumerary centrosomes, which may, in fact, reflect the formation of abnormal microtubule-based structures [21].

Several studies, carried out in human and Drosophila cell lines, have shown that PLK4 abundance at the centrosome fluctuates during the cell cycle $[11,18,19]$. In the case of Drosophila S2 cells, PLK4 is almost undetectable in interphase, but is clearly detectable during mitosis where its levels are at their highest $[18,19]$. In cultured human cells, PLK4 levels at centrosomes follow a similar trend, with levels being low in G1 and increasing incrementally from $S$ phase onwards to reach a maximum in mitosis [11]. While these data suggest that high levels of PLK4 are required during mitosis they do not give any indication of the kinase's activity. One study took advantage of the fact that PLK4, like many kinases, autophosphorylates upon activation [11]. Several potential autophosphorylation sites were identified and one of these, S305, was found to be phosphorylated in cultured cells. By raising a phospho-specific antibody against one of these sites, S305, it was possible to determine when PLK4 became active in the cell cycle. This revealed a number of remarkable findings. Firstly, PLK4 is present at centrioles in G1, but S305 phosphorylated PLK4 is undetectable suggesting that the kinase is inactive at this point in the cell cycle (Figure 3). PLK4 first becomes active in $S$ phase and the amount of active kinase approximately doubles at each cell cycle transition to reach a maximum in mitosis. Secondly, there is a delay in the activation of PLK4 at the replicating daughter centriole, with PLK4 first becoming active at the replicating mother centriole in $\mathrm{S}$ phase and then later at the replicating daughter centriole in G2. Thirdly, more active PLK4 is associated with the replicating mother centriole than the replicating daughter throughout interphase although by mitosis parity is reached. These results support the proposal that centriole may be initiated at the mother centriole first and then later at the daughter centriole. Lastly, it has been shown that active PLK4 is restricted to the centrosome, which may serve as a mechanism preventing centriole formation elsewhere in the cell and ensure that de novo centriole formation does not occur.

\section{The regulation of PLK4 stability}

PLK4 is a short-lived protein, with a half-life of between 2 to 3 hours, which is ubiquitinylated and degraded by the proteasome $[1,55]$. The stability of the kinase is governed by three PEST sequences [1], one within the amino-terminus and two within the carboxy terminus of the kinase, and internal deletion studies have shown that

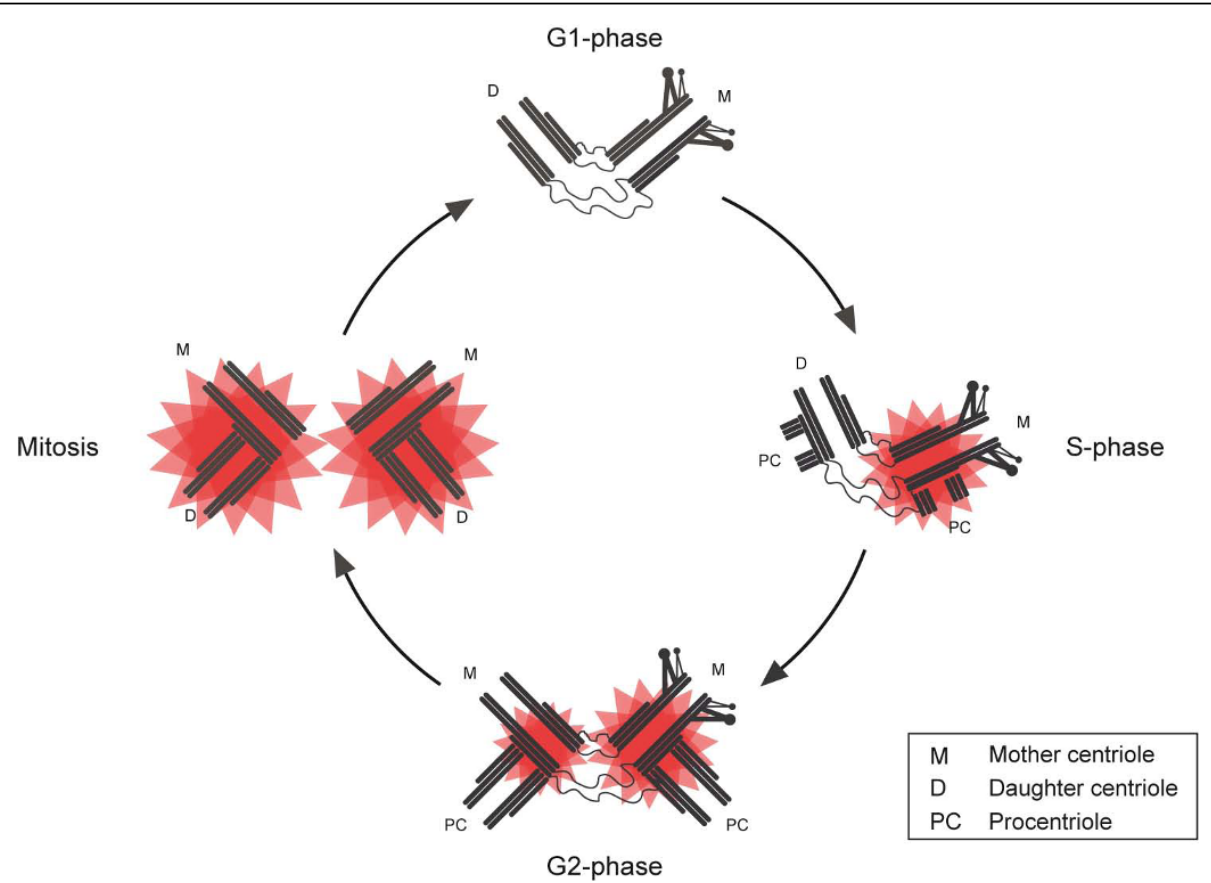

Figure 3 Centriole duplication and temporal activation of PLK4. In G1, PLK4 is present at centrioles but active kinase cannot be detected indicating that PLK4 is inactive at this point in the cell cycle. At the G1/S boundary centriole duplication begins with the formation of procentrioles at the proximal end of each parental centriole. This coincides with activation of PLK4 at the mother centriole (active kinase drawn as a red star). PLK4 becomes active at the replicating daughter centriole later on in the cell cycle in G2. By mitosis both centrosomes possess a similar amount of active PLK4 and procentriole elongation has been completed. 
all of them play a part in controlling the turn-over of the kinase [8]. Deletion of the first PEST sequence, however, stabilizes the kinase more than deletion of the two carboxy-terminal PEST sequences, suggesting that it has a greater influence in controlling PLK4 stability [8]. The first PEST sequence of PLK4 contains a degron motif, DGSXXT, which is conserved through evolution being present in $H$. sapiens, $M$. musculus, D. melanogaster, D. rerio and $X$. laevis PLK4 (Figure 4) [18,19]. Phosphorylation of the serine and threonine residues in the degron motif generates a binding site for the ubiquitin ligase complex, Skp1/cullin/F-box (SCF). The SCF consists of cullin 1 , a scaffolding protein that binds two invariable proteins Skp1, Rbx and a variable F-box protein, which determines the target specificity of the complex [56,57]. The SCF ubiquitin ligase complex has previously been implicated in centriole duplication. The Drosophila slimb ${ }^{\text {crd }}$ (centrosome replication defective) mutant, which has a P-element insert within the 5 ' untranslated region of slimb, a F-box protein, possesses supernumerary centrosomes although not all of the centrosomes that form are mature and able to nucleate microtubules [58]. There is also evidence indicating that mammalian slimb $(\beta$ $\operatorname{TrCP})$ is involved in regulating centriole duplication. Embryonic fibroblasts derived from $\beta-\mathrm{TrCP}-/$ - mice have an increased incidence of supernumerary centrosomes compared to wild-type MEFs although this may be due, in part, to a mitotic defect [59].

Upon binding to the phosphorylated degron motif the SCF ubiquitinates PLK4 and it is targeted to the proteasome for destruction $[18,19]$. Two different approaches were employed to demonstrate a specific role for the $\mathrm{SCF}^{\text {slimb/ } / \mathrm{T}-\mathrm{TrCP}}$ complex in regulating the turnover of
PLK4. The first involved disrupting the $\mathrm{SCF}^{\text {slimb/ } \beta-\operatorname{TrCP}}$ complex by siRNA-mediated depletion of either the cullin or slimb/ $\beta$-TrCP subunits. The second approach involved mutation of the serine and threonine residues within the degron motif of PLK4 to alanine, to prevent phosphorylation and generate a stabilized form of the

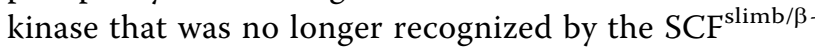
${ }^{T r C P}$ complex. Both of these approaches resulted in elevated levels of centriole amplification compared to control siRNA depletions or over-expression of the wild-type form PLK4. These results led to the proposal that the increased incidence of centriole amplification is directly attributable to the higher expression level of PLK4 $[18,19]$. Mutation of the degron motif to prevent phosphorylation clearly stabilizes PLK4, but fluorescence intensity measurements have shown that there is no difference in the amount of over-expressed PLK4 at the centrosomes of wild-type or degron-mutated PLK4transfected cells exhibiting centriole amplification. Furthermore, mutation of the degron motif appears to promote an increase in the amount of active PLK4 because a greater proportion of the kinase is S305 autophosphorylated [11]. These results suggest that the higher incidence of centriole amplification observed in cells expressing degron-mutated PLK4, compared to wildtype, is due to an increase in the amount of active kinase.

At present the kinase responsible for phosphorylating the degron motif is unknown but there is mounting evidence indicating that autophosphorylation plays a role in regulating the stability of the kinase. A link between PLK4 autophosphorylation and kinase stability was first established when it was observed that mutation of the kinase domain, to render the kinase catalytically inactive,
H. sapiens

M. musculus

D. melanogaster

D. rerio

$X$. laevis

\section{(P) P}

281 DSIDSGHATISTAITASSSTSISGSLFDKR 310 281 DSMDSGHATLSTTITASSGTSLSGSLLDRR 310 289 ESGDSGIITFASSDSRNSQQIRSVENSGPQ 318 282 GSIDSGIATISTASNATNNSSSSRLQRRTR 311 281 DSMDSGHATISTGFTGSSGVSISGRFQEKR 310

\section{Degron motif}

Figure 4 Regulatory phosphorylation sites in PLK4. The degron motif of PLK4 (highlighted in blue) is conserved with phosphorylation of its serine and threonine residues creating a binding site for the F-box protein $\beta$-TrCP, which forms part of the SCF $\beta$-TrCP ubiquitin ligase complex. Upon SCF $\beta$-TrCP binding, PLK4 is subsequently ubiquitinated and targeted to the proteasome for degradation. The identity of the kinase responsible for phosphorylating the two residues in the degron motif is currently unknown. Autophosphorylation plays a role in controlling the stability of PLK4 and it has been shown that the region spanning residues 282 to 305 of M. musculus PLK4 is heavily autophosphorylated. The precise identities of the residues autophosphorylated are not known and only potential sites can be proposed (marked in red). One of these sites, S305, is conserved and is autophosphorylated in H. sapiens PLK4 (marked in green), although it has no direct role in regulating the turnover of the kinase directly because its mutation to an alanine does not increase the stability of the kinase. However, it does seem to play a role in centriole duplication with its mutation to a glutamate increasing the incidence of centriole amplification in PLK4-overexpressing cells. 
resulted in increased expression of the kinase compared to wild-type [8]. A 23 amino acid region beyond the catalytic domain in mouse PLK4, which encompasses the degron motif, is heavily autophosphorylated and the deletion of this entire region vastly increases its stability and ability to trigger centriole amplification [60]. Similarly, mutation of the serine and threonine residues to alanine in this region stabilizes the kinase and augments its ability to amplify centriole number [60]. A more recent paper has elegantly shown that PLK4 autophosphorylation occurs in trans, where the molecules in the dimer phosphorylate each other, and confirmed that autophosphorylation is necessary to target the kinase for degradation [61]. This finding also explains why inducible cell lines stably transfected with catalytically inactive PLK4 are able to trigger centriole amplification upon induction of gene expression. Catalytically inactive PLK4 dimerizes with the endogenous kinase, but is unable to phosphorylate it, which effectively protects the endogenous kinase from degradation because the $\mathrm{SCF}^{\beta-\operatorname{TrCP}}$ complex cannot bind to it. As the endogenous kinase in no longer under the control of this ubiquitin-mediated degradation pathway centriole amplification ensues [61].

Where SCF-mediated ubiquitination takes place in the cell has yet to be determined conclusively. Components of the SCF including Skp1 and culllin 1 have been found localize to the centrosome throughout the cell cycle $[62,63]$. Cullin 1 seems to be enriched on the mother centriole [64], where there is more PLK4 and centriole duplication is probably first initiated [65], suggesting that there may be a greater demand for SCF activity at this site to prevent centriole amplification.

While it is clear that the $\mathrm{SCF}^{\beta-\mathrm{TrCP}}$ ubiquitin ligase complex has an important role in targeting PLK4 to the proteasome for degradation, it is not the only factor controlling the kinase's turn-over and stability. Drosophila PLK4 SCF ${ }^{\text {slimb }}$-binding mutants that can no longer be phosphorylated on the degron motif are still subject to degradation in G2 phase of the cell cycle [19]. The introduction of similar mutations in mouse PLK4 still results in degradation of the kinase. It is possible that in the absence of $\mathrm{SCF}^{\beta-\mathrm{TrCP}}$ activity the APC/C ubiquitin ligase complex may take over, as this ubiquitin ligase has been proposed to be involved in regulating PLK4 degradation before [66].

\section{Alternative forms of regulation}

The stability of PLK4 may be governed by alternative mechanisms such as the phosphorylation-dependent stabilization of PLK4 by other kinases and there are data to suggest this is the case. Yamashita et al demonstrated that phosphorylation of a tyrosine residue in the N-terminus of PLK4 by the kinase Tec increased the stability of PLK4 and promoted PLK4 autophosphorylation [8]. The Tec-dependent increase in PLK4 stability is interesting because it suggests that phosphorylation by other kinases may play a role in governing its turn-over. Such a mechanism might be at work at centrosome and it could result in the local stabilization of PLK4 at this site. In support of this it has been shown that S305 autophosphorylated PLK4 at centrosomes exhibits a similar shift in mobility as Tec phosphorylated PLK4 [11].

Another possible regulatory mechanism could be proposed from work carried out on the C. elegans centrosomal kinase, zyg-1. In a screen for suppressors of the zyg1(it25) temperature-sensitive mutant allele a number of candidate genes were identified including suppressor of zyg-1 $20(s z y-20)[67,68]$. This gene encodes an RNAbinding protein that localizes to the centrosome and appears to negatively regulate the abundance of zyg-1 [68]. Szy-20 is a conserved and it will interesting to see if the vertebrate homologues of this protein are involved in controlling PLK4 abundance at centrosomes.

\section{Transcriptional control of PLK4}

While much attention has been focused on proteasomemediated degradation of PLK4 it is important not to overlook the fact that the PLK4 gene is transcribed in a cell cycle-dependent manner. PLK4 transcript levels are undetectable in G0, low in G1 and progressively increase through $\mathrm{S}$ and $\mathrm{G} 2$ to reach a maximum in mitosis [66]. It seems that PLK4 protein levels mirror those of PLK4 mRNA suggesting that gene transcription has a significant impact on controlling the overall expression level of PLK4. At present, little is known about the transcription factors controlling expression of the PLK4 gene. One report has shown that expression of the human PLK4 gene can be suppressed by the tumour suppressor p53 and this is dependent upon the activity of histone deacetylases (HDACs) [29].

\section{Conclusions}

PLK1, PLK2 and PLK4 act in concert to control the licensing and duplication of centrioles and centrosome maturation. PLK4 represents a separate branch of the PLK family because it shares little homology with its other members as a result of rapid divergence through evolution. Its function in controlling centriole/basal body duplication is a result of sub-functionalization after duplication of the PLK gene. Before the innovation of PLK4, basal body duplication was probably under the control of a single PLK, although there are some ciliated species that do not have a PLK gene.

PLK4's role in centriole duplication is essential yet many questions remain to be answered. At present, no centriolar PLK4 substrates have been identified although one possible substrate is SAS-6, as work in C. elegans has shown that this protein is phosphorylated by zyg- 1 . The identification of PLK4 substrates should help to 
delineate the centriole duplication pathway and determine whether the kinase acts just once, to initiate duplication, or at multiple stages during the duplication process. It will also be important to identify the protein responsible for anchoring PLK4 to the centrosome via its crypto Polo and Polo-box domains. As PLK4 localizes to the proximal ends of and along the walls of centrioles it seems likely that PLK4 will interact with multiple proteins at the centrosome.

Determining how $\mathrm{SCF}^{\beta-\operatorname{TrCP}}$ ubiquitin ligase-mediated degradation of PLK4 is coordinated and influenced by other factors during the cell cycle is crucial to understand how PLK4 levels are maintained within a certain threshold during the cell cycle. Clearly, if the threshold is crossed and PLK4 levels rise above normal the consequences can be catastrophic, particularly during centriole duplication because it overrides the licensing mechanism and multiple procentrioles form at each parental centriole.

The recent discovery that PLK4 is involved in cytokinetic exit broadens the role of this kinase beyond centriole duplication and demonstrates that the kinase has multiple functions in the cell. Several lines of evidence support a role for PLK4 in mitotic progression, including the identification of mitotic substrates such as CDC25C and Ect2, the delayed entry of PLK4+/- dividing hepatocytes into mitosis coupled with persistently elevated levels of cyclin B1 and the fact that active PLK4 levels reach a maximum during mitosis. This suggests PLK4 is linked to cell cycle regulators, but also raises the possibility that the kinase is involved in regulating centrosome maturation, such as procentriole elongation, which is completed in early mitosis, or the transformation of the daughter centriole into a mother centriole (appendage formation). It is clear that much work remains to be done before we fully understand the functions of this kinase in the cell.

\section{Authors' contributions}

JES made the figures and drafted the manuscript. MB read and corrected the manuscript. All authors read and approved the final manuscript.

\section{Competing interests}

The authors declare that they have no competing interests.

Received: 27 August 2010 Accepted: 29 September 2010 Published: 29 September 2010

\section{References}

1. Fode C, Motro B, Yousefi S, Heffernan M, Dennis JW: Sak, a murine protein-serine/threonine kinase that is related to the Drosophila polo kinase and involved in cell proliferation. Proceedings of the National Academy of Sciences of the United States of America 1994, 91(14):6388-6392.

2. Carvalho-Santos Z, Machado P, Branco P, Tavares-Cadete F, RodriguesMartins A, Pereira-Leal JB, Bettencourt-Dias M: Stepwise evolution of the centriole-assembly pathway. Journal of cell science 2010, 123(Pt 9):1414-1426.

3. Hodges ME, Scheumann N, Wickstead B, Langdale JA, Gull K Reconstructing the evolutionary history of the centriole from protein components. Journal of cell science 2010, 123(Pt 9):1407-1413.
4. O'Connell KF, Caron C, Kopish KR, Hurd DD, Kemphues KJ, Li Y, White JG: The $C$. elegans zyg-1 gene encodes a regulator of centrosome duplication with distinct maternal and paternal roles in the embryo. Cell 2001, 105(4):547-558.

5. Lowery DM, Lim D, Yaffe MB: Structure and function of Polo-like kinases. Oncogene 2005, 24(2):248-259.

6. Donohue PJ, Alberts GF, Guo Y, Winkles JA: Identification by targeted differential display of an immediate early gene encoding a putative serine/threonine kinase. The Journal of biological chemistry 1995, 270(17):10351-10357.

7. Simmons DL, Neel BG, Stevens R, Evett G, Erikson RL: Identification of an early-growth-response gene encoding a novel putative protein kinase. Molecular and cellular biology 1992, 12(9):4164-4169.

8. Yamashita Y, Kajigaya S, Yoshida K, Ueno S, Ota J, Ohmine K, Ueda M, Miyazato A, Ohya K, Kitamura T, et al: Sak serine-threonine kinase acts as an effector of Tec tyrosine kinase. The Journal of biological chemistry 2001, 276(42):39012-39020

9. Johnson EF, Stewart KD, Woods KW, Giranda VL, Luo Y: Pharmacological and functional comparison of the polo-like kinase family: insight into inhibitor and substrate specificity. Biochemistry 2007, 46(33):9551-9563.

10. Leung GC, Ho CS, Blasutig IM, Murphy JM, Sicheri F: Determination of the Plk4/Sak consensus phosphorylation motif using peptide spots arrays. FEBS letters 2007, 581(1):77-83.

11. Sillibourne JE, Tack F, Vloemans N, Boeckx A, Thambirajah S, Bonnet $P$ Ramaekers FC, Bornens M, Grand-Perret T: Autophosphorylation of pololike kinase 4 and its role in centriole duplication. Molecular biology of the cell 2010, 21(4):547-561.

12. Elia $A E$, Cantley LC, Yaffe MB: Proteomic screen finds pSer/pThr-binding domain localizing Plk1 to mitotic substrates. Science (New York, NY) 2003, 299(5610):1228-1231.

13. Elia AE, Rellos P, Haire LF, Chao JW, Ivins FJ, Hoepker K, Mohammad D, Cantley LC, Smerdon SJ, Yaffe MB: The molecular basis for phosphodependent substrate targeting and regulation of Plks by the Polo-box domain. Cell 2003, 115(1):83-95.

14. Leung GC, Hudson JW, Kozarova A, Davidson A, Dennis JW, Sicheri F: The Sak polo-box comprises a structural domain sufficient for mitotic subcellular localization. Nature structural biology 2002, 9(10):719-724.

15. Swallow CJ, Ko MA, Siddiqui NU, Hudson JW, Dennis JW: Sak/PIk4 and mitotic fidelity. Oncogene 2005, 24(2):306-312

16. Habedanck R, Stierhof YD, Wilkinson CJ, Nigg EA: The Polo kinase Plk4 functions in centriole duplication. Nature cell biology 2005, 7(11):1140-1146

17. Rechsteiner M, Rogers SW: PEST sequences and regulation by proteolysis. Trends in biochemical sciences 1996, 21(7):267-271.

18. Cunha-Ferreira I, Rodrigues-Martins A, Bento I, Riparbelli M, Zhang W, Laue E, Callaini G, Glover DM, Bettencourt-Dias M: The SCF/Slimb ubiquitin ligase limits centrosome amplification through degradation of SAK/PLK4. Curr Biol 2009, 19(1):43-49.

19. Rogers GC, Rusan NM, Roberts DM, Peifer M, Rogers SL: The SCF Slimb ubiquitin ligase regulates Plk4/Sak levels to block centriole reduplication. The Journal of cell biology 2009, 184(2):225-239.

20. Hudson JW, Kozarova A, Cheung P, Macmillan JC, Swallow CJ, Cross JC, Dennis JW: Late mitotic failure in mice lacking Sak, a polo-like kinase. Curr Biol 2001, 11(6):441-446.

21. Ko MA, Rosario CO, Hudson JW, Kulkarni S, Pollett A, Dennis JW, Swallow CJ: Plk4 haploinsufficiency causes mitotic infidelity and carcinogenesis. Nature genetics 2005, 37(8):883-888.

22. Bonni S, Ganuelas ML, Petrinac S, Hudson JW: Human Plk4 phosphorylates Cdc25C. Cell cycle (Georgetown, Tex) 2008, 7(4):545-547.

23. Rosario CO, Ko MA, Haffani YZ, Gladdy RA, Paderova J, Pollett A, Squire JA, Dennis JW, Swallow CJ: Plk4 is required for cytokinesis and maintenance of chromosomal stability. Proceedings of the National Academy of Sciences of the United States of America 2010, 107(15):6888-6893.

24. Wolfe BA, Takaki T, Petronczki M, Glotzer M: Polo-like kinase 1 directs assembly of the HsCyk-4 RhoGAP/Ect2 RhoGEF complex to initiate cleavage furrow formation. PLOS biology 2009, 7(5):e1000110.

25. Yuce O, Piekny A, Glotzer M: An ECT2-centralspindlin complex regulates the localization and function of RhoA. The Journal of cell biology 2005, 170(4):571-582.

26. Robbins $E$, Jentzsch $G$, Micali A: The centriole cycle in synchronized HeLa cells. The Journal of cell biology 1968, 36(2):329-339. 
27. Bettencourt-Dias M, Rodrigues-Martins A, Carpenter L, Riparbelli M, Lehmann L, Gatt MK, Carmo N, Balloux F, Callaini G, Glover DM: SAK/PLK4 is required for centriole duplication and flagella development. Curr Biol 2005, 15(24):2199-2207.

28. Rodrigues-Martins A, Riparbelli M, Callaini G, Glover DM, Bettencourt-Dias M: Revisiting the role of the mother centriole in centriole biogenesis. Science (New York, NY) 2007, 316(5827):1046-1050.

29. Li J, Tan M, Li L, Pamarthy D, Lawrence TS, Sun Y: SAK, a new polo-like kinase, is transcriptionally repressed by $\mathrm{p} 53$ and induces apoptosis upon RNAi silencing. Neoplasia (New York, NY) 2005, 7(4):312-323.

30. Chretien D, Buendia B, Fuller SD, Karsenti E: Reconstruction of the centrosome cycle from cryoelectron micrographs. Journal of structural biology 1997, 120(2):117-133.

31. Paintrand M, Moudjou M, Delacroix H, Bornens M: Centrosome organization and centriole architecture: their sensitivity to divalent cations. Journal of structural biology 1992, 108(2):107-128.

32. Vorobjev IA, Chentsov Yu S: Centrioles in the cell cycle. I. Epithelial cells. The Journal of cell biology 1982, 93(3):938-949.

33. Loncarek J, Hergert P, Magidson V, Khodjakov A: Control of daughter centriole formation by the pericentriolar material. Nature cell biology 2008, 10(3):322-328.

34. Kuriyama R, Borisy GG: Centriole cycle in Chinese hamster ovary cells as determined by whole-mount electron microscopy. The Journal of cell biology 1981, 91(3 Pt 1):814-821.

35. Tsou MF, Stearns T: Mechanism limiting centrosome duplication to once per cell cycle. Nature 2006, 442(7105):947-951

36. Wong C, Stearns T: Centrosome number is controlled by a centrosomeintrinsic block to reduplication. Nature cell biology 2003, 5(6):539-544.

37. Rattner JB, Phillips SG: Independence of centriole formation and DNA synthesis. The Journal of cell biology 1973, 57(2):359-372.

38. Leidel S, Delattre M, Cerutti L, Baumer K, Gonczy P: SAS-6 defines a protein family required for centrosome duplication in $C$. elegans and in human cells. Nature cell biology 2005, 7(2):115-125.

39. Hiraki M, Nakazawa Y, Kamiya R, Hirono M: Bld10p constitutes the cartwheel-spoke tip and stabilizes the 9-fold symmetry of the centriole. Curr Biol 2007, 17(20):1778-1783.

40. Mottier-Pavie V, Megraw TL: Drosophila bld10 is a centriolar protein that regulates centriole, basal body, and motile cilium assembly. Molecular biology of the cell 2009, 20(10):2605-2614.

41. Dammermann A, Maddox PS, Desai A, Oegema K: SAS-4 is recruited to a dynamic structure in newly forming centrioles that is stabilized by the gamma-tubulin-mediated addition of centriolar microtubules. The Journal of cell biology 2008, 180(4):771-785.

42. Leidel S, Gonczy P: SAS-4 is essential for centrosome duplication in C elegans and is recruited to daughter centrioles once per cell cycle. Developmental cell 2003, 4(3):431-439.

43. Kleylein-Sohn J, Westendorf J, Le Clech M, Habedanck R, Stierhof YD, Nigg EA: Plk4-induced centriole biogenesis in human cells. Developmental cell 2007, 13(2):190-202.

44. Chen Z, Indjeian VB, McManus M, Wang L, Dynlacht BD: CP110, a cell cycle-dependent CDK substrate, regulates centrosome duplication in human cells. Developmental cell 2002, 3(3):339-350.

45. Kitagawa D, Busso C, Fluckiger I, Gonczy P: Phosphorylation of SAS-6 by ZYG-1 is critical for centriole formation in C. elegans embryos. Developmental cell 2009, 17(6):900-907.

46. Tsou MF, Wang WJ, George KA, Uryu K, Stearns T, Jallepalli PV: Polo kinase and separase regulate the mitotic licensing of centriole duplication in human cells. Developmental cell 2009, 17(3):344-354

47. Warnke S, Kemmler S, Hames RS, Tsai HL, Hoffmann-Rohrer U, Fry AM, Hoffmann I: Polo-like kinase-2 is required for centriole duplication in mammalian cells. Curr Biol 2004, 14(13):1200-1207.

48. Lacey KR, Jackson PK, Stearns T: Cyclin-dependent kinase control of centrosome duplication. Proceedings of the National Academy of Sciences of the United States of America 1999, 96(6):2817-2822.

49. Meraldi P, Lukas J, Fry AM, Bartek J, Nigg EA: Centrosome duplication in mammalian somatic cells requires E2F and Cdk2-cyclin A. Nature cell biology 1999, 1(2):88-93.

50. Fisk HA, Mattison CP, Winey M: Human Mps1 protein kinase is required for centrosome duplication and normal mitotic progression. Proceedings of the National Academy of Sciences of the United States of America 2003, 100(25):14875-14880.
51. Wu J, Cho HP, Rhee DB, Johnson DK, Dunlap J, Liu Y, Wang Y: Cdc14B depletion leads to centriole amplification, and its overexpression prevents unscheduled centriole duplication. The Journal of cell biology 2008, 181(3):475-483

52. Azimzadeh J, Hergert P, Delouvee A, Euteneuer U, Formstecher E, Khodjakov A, Bornens M: hPOC5 is a centrin-binding protein required for assembly of full-length centrioles. The Journal of cell biology 2009, 185(1):101-114.

53. Piel M, Nordberg J, Euteneuer U, Bornens M: Centrosome-dependent exit of cytokinesis in animal cells. Science (New York, NY) 2001 291(5508):1550-1553.

54. Kuriyama R, Bettencourt-Dias M, Hoffmann I, Arnold M, Sandvig L: Gammatubulin-containing abnormal centrioles are induced by insufficient Plk4 in human HCT116 colorectal cancer cells. Journal of cell science 2009, 122(Pt 12):2014-2023.

55. Duensing A, Liu Y, Perdreau SA, Kleylein-Sohn J, Nigg EA, Duensing S: Centriole overduplication through the concurrent formation of multiple daughter centrioles at single maternal templates. Oncogene 2007, 26(43):6280-6288.

56. Cenciarelli C, Chiaur DS, Guardavaccaro D, Parks W, Vidal M, Pagano M: Identification of a family of human F-box proteins. Curr Biol 1999, 9(20):1177-1179.

57. Kipreos ET, Pagano M: The F-box protein family. Genome biology 2000 1(5):REVIEWS3002.

58. Wojcik EJ, Glover DM, Hays TS: The SCF ubiquitin ligase protein slimb regulates centrosome duplication in Drosophila. Curr Biol 2000, 10(18):1131-1134.

59. Guardavaccaro D, Kudo Y, Boulaire J, Barchi M, Busino L, Donzelli M, Margottin-Goguet F, Jackson PK, Yamasaki L, Pagano M: Control of meiotic and mitotic progression by the $\mathrm{F}$ box protein beta-Trcp1 in vivo. Developmental cell 2003, 4(6):799-812.

60. Holland AJ, Lan W, Niessen S, Hoover H, Cleveland DW: Polo-like kinase 4 kinase activity limits centrosome overduplication by autoregulating its own stability. The Journal of cell biology 2010, 188(2):191-198.

61. Guderian G, Westendorf J, Uldschmid A, Nigg EA: Plk4 transautophosphorylation regulates centriole number by controlling betaTrCP-mediated degradation. Journal of cell science 2010, 123(Pt 13):2163-2169.

62. Freed E, Lacey KR, Huie P, Lyapina SA, Deshaies RJ, Stearns T, Jackson PK: Components of an SCF ubiquitin ligase localize to the centrosome and regulate the centrosome duplication cycle. Genes \& development 1999 13(17):2242-2257.

63. Gstaiger M, Marti A, Krek W: Association of human SCF(SKP2) subunit p19 (SKP1) with interphase centrosomes and mitotic spindle poles. Experimental cell research 1999, 247(2):554-562

64. Korzeniewski N, Zheng L, Cuevas R, Parry J, Chatterjee P, Anderton B, Duensing A, Munger $K$, Duensing S: Cullin 1 functions as a centrosomal suppressor of centriole multiplication by regulating polo-like kinase 4 protein levels. Cancer research 2009, 69(16):6668-6675.

65. White RA, Pan Z, Salisbury JL: GFP-centrin as a marker for centriole dynamics in living cells. Microscopy research and technique 2000, 49(5):451-457.

66. Fode C, Binkert C, Dennis JW: Constitutive expression of murine Sak-a suppresses cell growth and induces multinucleation. Molecular and cellular biology 1996, 16(9):4665-4672.

67. Kemp CA, Song MH, Addepalli MK, Hunter G, O'Connell K: Suppressors of zyg-1 define regulators of centrosome duplication and nuclear association in Caenorhabditis elegans. Genetics 2007, 176(1):95-113.

68. Song MH, Aravind L, Muller-Reichert T, O'Connell KF: The conserved protein SZY-20 opposes the Plk4-related kinase ZYG-1 to limit centrosome size. Developmental cell 2008, 15(6):901-912

doi:10.1186/1747-1028-5-25

Cite this article as: Sillibourne and Bornens: Polo-like kinase 4: the odd one out of the family. Cell Division 2010 5:25. 